\title{
Telecardiology under Resource Constraint: Low-complexity Compact Representation of ECG
}

\author{
Roopak R Tamboli, Soumya Jana
}

Indian Institute of Technology Hyderabad, Telangana, India

\begin{abstract}
Compact, yet faithful, representation of ECG signals to meet bandwidth and power constraints remains central to successful telecardiology, primarily in infrastructuredeficient areas. Towards practical realization, we seek desired compactness in the class of low-complexity transform representations. A typical ECG signal consists of a strong rhythmic (low-pass) component, with compact Fourier transform representation, and temporally localized (high-pass) features, efficiently represented by wavelet transform. Accordingly, we propose a compact representation consisting of suitable Fourier and wavelet coefficients. As computation of such coefficients is at most $O(n \log n)$, the proposed representation inherits the desired low complexity. Our method achieves targeted representation accuracy using fewer transform coefficients compared to the well-known fixed transform representations.
\end{abstract}

\section{Introduction}

Electrocardiogram (ECG) is an indispensable tool in monitoring and management of cardiac health. ECG records are increasingly being maintained in electronic form, and machines directly producing digitized ECG signals are now commonplace [1]. Very often, such signals are stored locally, or transmitted to a remote location, respectively, for decision making at a later point, or at a distance [2]. Accordingly, with a view to minimizing storage requirement and/or communication bandwidth, one desires to represent ECG signals as compactly as possible without adversely affecting eventual clinical interpretation.

Interestingly, the problem of practical ECG compression has been studied since several decades. Efficacy of a compression algorithm depends on signal sparsity. In this context, various researchers have reported ECG signals to be sparse in wavelet bases, and in particular "Daubechies 4" (db4) wavelet basis [3,4]. In the process, various researchers observed signal sparsity in wavelet and related domains, and demonstrated the respective efficacy of discrete cosine transform (DCT) [5], wavelet packets [6],

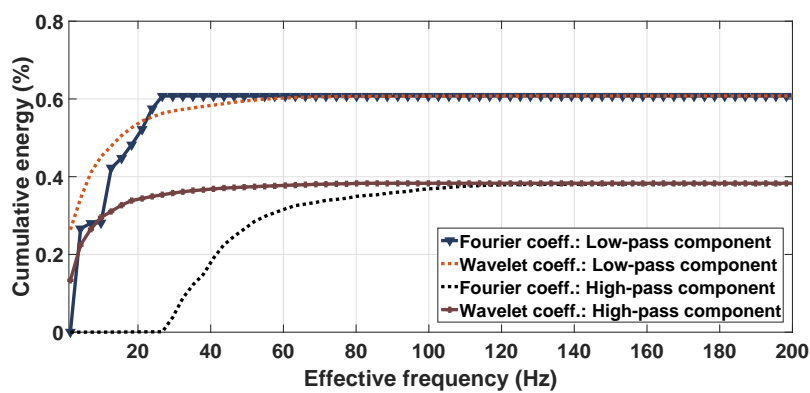

Figure 1: Cumulative energy plot for a segment of record '100': Comparison of Fourier and wavelet techniques in terms of ECG energy packing.

SPIHT (set partitioning in hierarchical trees) algorithm [7]. Signal sparsity is also central to compressed sensing of ECG signals [3]. Moreover, in denoising applications, a sparser representation allows more coefficients to fall below a threshold thus allowing more noise to be removed [8].

In an earlier work, we proposed a Hybrid Fourier/wavelet technique for ECG signal approximation [9]. The method was demonstrated on signals derived from ANSI/AAMI EC13 dataset [10]. In this paper, we present a comparative study of ECG signal representation using Fourier transform (FT), wavelet transform (WT), discrete cosine transform (DCT) and the proposed hybrid method. The signals for the experimental dataset were picked from MITBIH Arrhythmia database [10]. Compared to the DCT, FT and WT representations, the proposed method saves of $46.15 \%, 25 \%$ and $4.54 \%$ coefficients on average, for certain target representation accuracy, respectively.

The rest of the paper is organized as follows. We begin with describing theoretical background and the key idea in Sec. 2. The proposed hybrid encoder is briefly described in Sec. 2.1. Details of experimental data generation are provided in Sec. 3. The results of signal approximation are presented in Sec. 4. We conclude with a discussion in Sec. 5 . 


\section{Key Idea}

We begin with mathematical description of the ECG signal approximation problem. Consider set $\mathcal{E}$ of ECG signals of length $N$. Intuitively, $\mathcal{E}$ should be a relatively small subset of the set $\mathcal{R}^{\mathcal{N}}$ of all $N$-length signals. In the linear signal approximation problem, one assumes the existence of subspace $\mathcal{S}$ of dimension $K<<N$ such that projection $\hat{x}$ of any $x \in \mathcal{E}$ onto $\mathcal{S}$ provides an $\epsilon$-accurate linear approximation, i.e., $\|x-\hat{x}\|<\epsilon\|x\|$ for small $\epsilon>0$. Here $\|\cdot\|$ indicates norm generally, and the 2 -norm specifically. One then seeks that subspace which achieves $\epsilon$-accuracy with minimum $K$. In transform coding parlance, the above translates to the problem of identifying the optimal unitary transform $U$ such that the minimum number $K$ of transform coefficients provides $\epsilon$-accuracy. In this framework, the same transform $U$ is applied to each signal $x \in \mathcal{E}$, and the locations of the preserved coefficients are independent of $x$.

In a hypothetical scenario where ECG signals admit a statistical model, one would view various observed signals as realizations of an underlying random vector $X \in \mathcal{R}^{\mathcal{N}}$. In addition, if $X$ were Gaussian, the optimal transform is known to be the Karhunen-Loève transform (KLT). Further, assuming the KLT coefficients are arranged in the descending order, one would keep the first $K$ coefficients such that their energy is within a factor $\epsilon$ of the aggregate signal energy [5]. However, practicality of aforementioned statistical techniques still remains problematic, as numerous heart conditions, both normal and abnormal, bearing specific temporal signatures arise, making comprehensive statistical modeling of ECG signals difficult.

In this backdrop, we take a closer look at the structure of ECG signals, which consists of a strong rhythmic (low-pass) component, and various temporally localized features (high-pass component). The former contains the significant part of signal energy. Specifically, Fig. 1 depicts a conservative example, where $90 \%$ of the signal energy is packed only up to a frequency of about 30 Hz. Interestingly, although unsuitable for encoding the entire spectrum, Fourier representation remains efficient for encoding such low-pass component. On the other hand, the the residual high-pass component accounts for temporally localized features that are compactly represented by wavelets. Clearly, the latter component would require a far larger number of coefficients in a Fourier representation. In view of the this observation, we propose an encoding algorithm that divides the ECG spectrum into low-pass and high-pass components, and use the Fourier and the wavelet coefficients respectively for their encoding. In this paper, we make use of the 'db4' wavelet basis in view of its reported superiority [3].

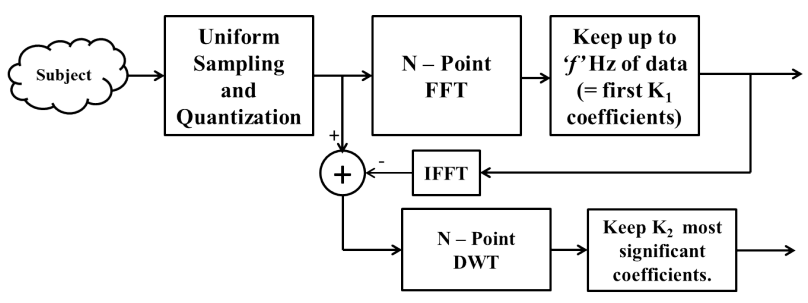

Figure 2: Conceptual block diagram of hybrid Fourier/wavelet encoder.

\subsection{Hybrid Fourier/Wavelet Encoder}

The proposed encoder, depicted in Fig. 2, is parameterized by three integers $N, K_{1}$ and $K_{2}$. Specifically, $N$ denotes the signal length. Further, the first $K_{1}$ coefficients of the $N$-point FFT of the signal are retained, which amounts to $2 K_{1}$ numbers. Thus the parameter $K_{1}$ determines the cutoff frequency for the low-pass components. Next, the corresponding low-pass signal, obtained via $N$-point inverse FFT (IFFT), is subtracted from the original, and the residual high-pass component remains. We take $N$-point discrete wavelet transform (DWT) of that residual, and retain $K_{2}$ most significant coefficients. However, such significant coefficients are expected to occur at isolated locations, which we also need to preserve. As a result, we end up with $2 K_{2}$ numbers. For the sake of simplicity, each number is assumed to be represented by a machine word, whose length is not optimized. In this setup, the optimal pair $\left(K_{1}, K_{2}\right)$ is sought such that the total number of retained numbers $2\left(K_{1}+K_{2}\right)$ is minimized such that the worst-case approximation over all ECG signals is above a certain threshold.

\section{Experimental Details}

We considered 43 annotated ECG records of the MITBIH Arrhythmia database [10]. The MIT-BIH Arrhythmia Database contains 48 half-hour excerpts of two-channel ambulatory ECG recordings. The records are digitized at $360 \mathrm{~Hz}$ per channel with $11-$ bit resolution. The primary reasons for choosing this dataset include: (i) wide popularity in the literature, (ii) availability of annotations, and (iii) variety of arrhythmias.

From the first channel of those records, overlapping signal vectors of length 256 samples, each containing an Rpeak, were formed. As explained in Sec. 2.1, we then optimized the pair $\left(K_{1}, K_{2}\right)$, where $K_{1}$ Fourier coefficients and $K_{2}$ wavelet (Daubechies-4, 'db4') coefficients respectively represent the rhythmic component and localized features, and jointly achieve a target fidelity. The number, $K_{1}+K_{2}$, of coefficients in the proposed method was compared to that required to achieve the same target fidelity 


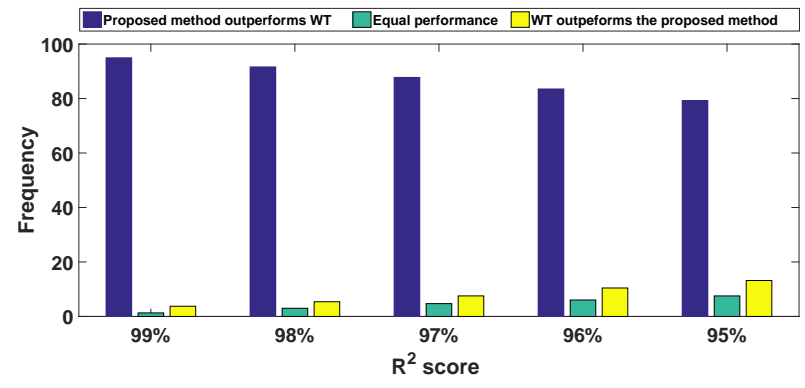

Figure 3: Histogram for performace comparison.

Table 1: \% savings offerred by the proposed method

\begin{tabular}{cccccc} 
& \multicolumn{5}{c}{ Targetted $R^{2}$ score (in \%) } \\
\cline { 2 - 6 } & 99 & 98 & 97 & 96 & 95 \\
\hline DCT & 46.15 & 51.52 & 53.33 & 53.57 & 53.85 \\
FT & 25.00 & 27.27 & 30.00 & 31.58 & 33.33 \\
WT & 4.54 & 11.11 & 6.67 & 7.14 & 7.69 \\
\hline
\end{tabular}

using (i) wavelet transform (WT) ('db4'), (ii) discrete cosine transform (DCT), and (iii) Fourier transform (FT).

The fidelity of representation was measured using $R^{2}$ score, defined as,

$$
R^{2}=1-\frac{\|x-\hat{x}\|_{2}^{2}}{\|x-\bar{x}\|_{2}{ }^{2}} .
$$

Here, $x$ and $\hat{x}$ denote the original signal and its approximation, respectively. $\bar{x}$ denotes the mean value of the signal $x$. The score is generally presented as a percentage.

\section{Results}

We now present the performance of the proposed method in terms of compactness of representation and associated error, at certain target fidelities. Fig. 4 shows the superiority of the proposed method for individual records. In order to achieve desired accuracy, the proposed method requires fewer number of coefficients across all the 43 signals. For any ECG record, the number of coefficients depicted is obtained by averaging over all signal segments derived from that record. The efficacy of the proposed method is pronounced for the records that are difficult to compress (observe the peaks in Fig. 4). Compared to DCT, FT and WT representations, respectively, the proposed method saves $46.15 \%, 25 \%$ and $4.54 \%$ coefficients on the average, at a target fidelity $R^{2}=99 \%$. The comparison for various target $R^{2}$ scores are tabulated in Table 1 . Since the difference between average number coefficients for the hybrid method and WT-based representation is less, the \% saving does not increase uniformly with decreasing target fidelities. In Fig. 3 we show that the proposed
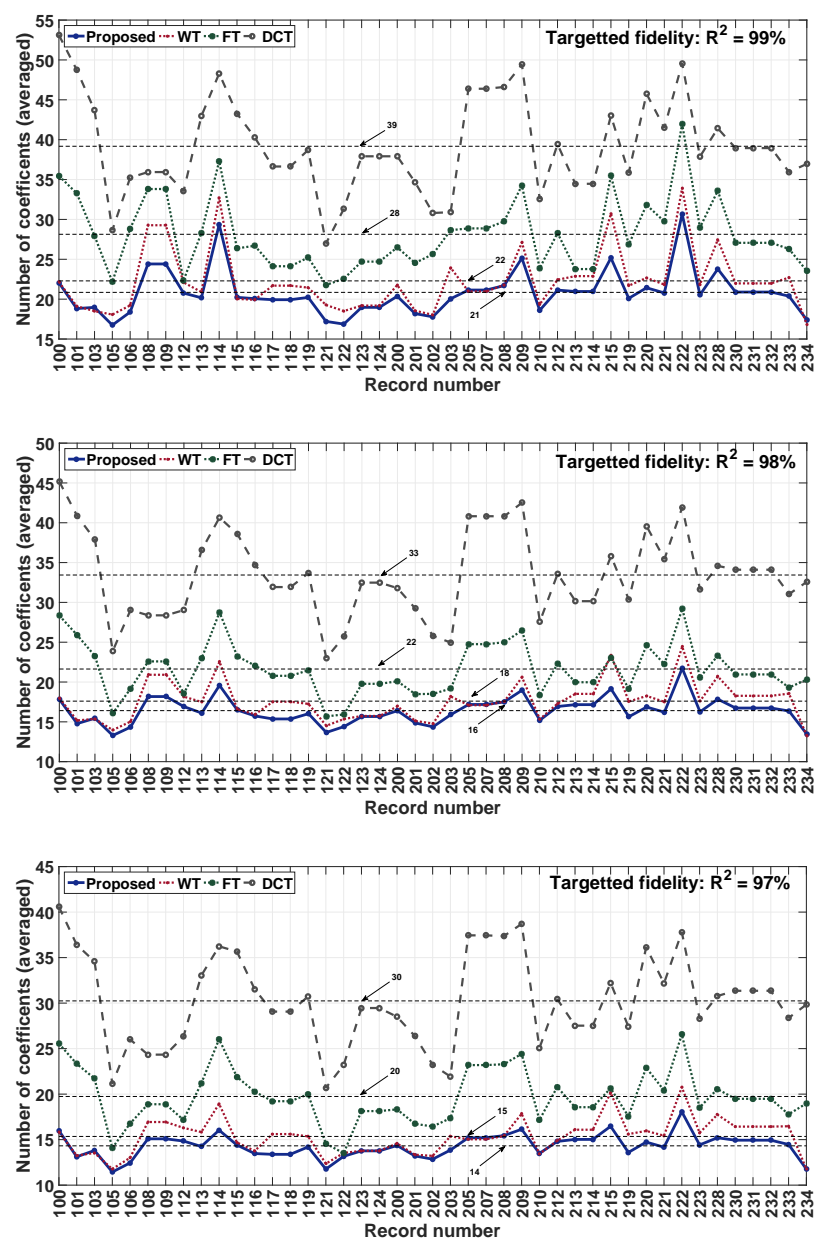

Figure 4: Performance of the proposed approximation method at various target fidelities

method performs better than or same as (for lower target fidelities) the wavelet representation for over $90 \%$ of the tested signals. Interestingly, for target fidelity of $R^{2}=99 \%$, the proposed method clearly dominates over wavelet representation.

Next, we present approximation a particular record along with the associated errors. For this purpose, we chose the least compressible signal in the dataset, namely the record '100' (see Fig. 4). For ease of understating and visualization, we chose first 4096 samples of the first channel from record ' 100 '. We then approximated this signal of length 4096 using FT-, WT- and DCT-based representation as well as the proposed method. The target fidelity was set at $R^{2}=99 \%$. While the approximated signals in Figs. 5(b), 5(d), 5(f) and 5(h) appear similar, the approximation errors shown in Figs. 5(c), 5(e), 5(g) and 5(i) reveal the difference in performance of various methods. The FTand DCT-based representations suffer from error accumulation at feature points. The wavelet and hybrid strategies, 


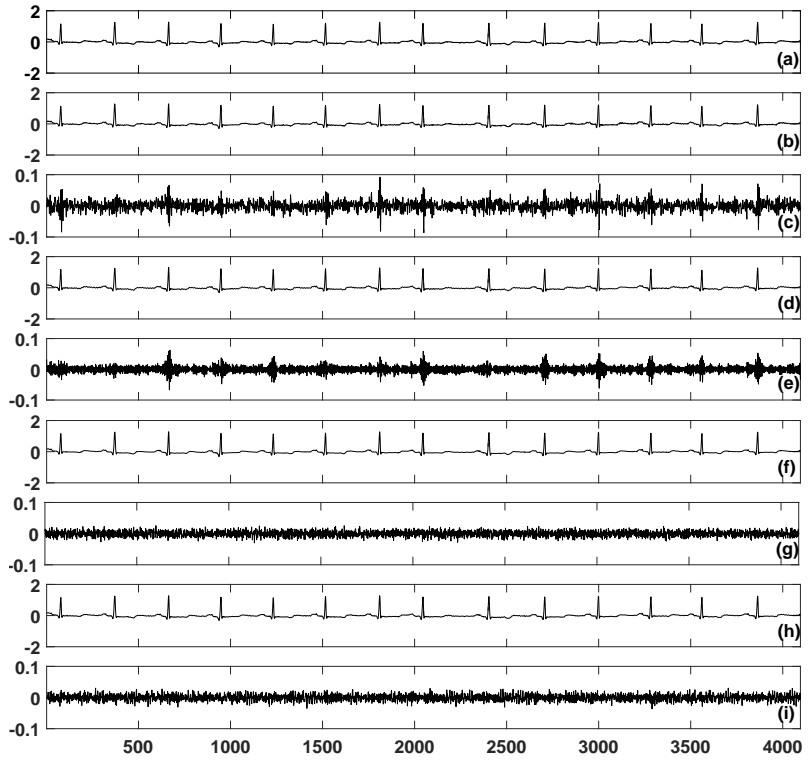

Figure 5: Representation of a segment of record '100' at $R^{2}=99 \%$. (a) Original signal, (b) DCT, (d) FT, (f) WT and (h) Proposed method. Errors in these representations are shown in (c), (e), (g) and (i), respectively.

which are virtually indistinguishable, appear to spread the error more evenly. In Fig. 6, histograms of those errors are shown. As expected, the proposed method along with the wavelet representation exhibit a tall and fast-decaying histogram; i. e., errors of only minute magnitudes appear more frequently. On the contrary, in DCT and FT representations errors of significant magnitudes appear more frequently, resulting in heavy-tailed histogram.

\section{Discussion}

In summary, the proposed hybrid representation method proves to be more efficient than the state-of-the-art single transform-based techniques, without sacrificing the desirable error characteristics. It provides more dramatic savings over FT- or DCT-based representation. Moreover, such improved efficiency is achieved without sacrificing low complexity. Here, note that Karhunen Loève transform (KLT) provides the best compaction, albeit, at a higher complexity.

Our study exhaustively evaluates the proposed method over a large dataset containing several disease conditions and benchmarks the ECG representation at a specified error. Such a study assumes significance in the context of basis selection for various ECG signal processing applications, including compression, denoising and compressive sensing.

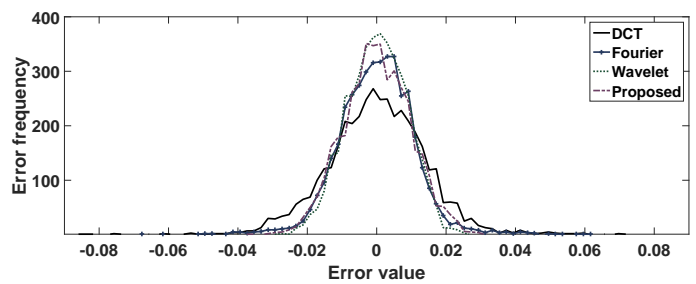

Figure 6: Error characteristics of various representation schemes at $R^{2}=99 \%$, for a segment of record ' 100 '.

\section{References}

[1] Srnmo L, Laguna P. Bioelectrical signal processing in cardiac and neurological applications. Academic Press; 2005.

[2] Chandra, Bollepalli S., Challa S. Sastry, and Soumya Jana. "Reliable resource-constrained telecardiology via compressive detection of anomalous ECG signals." Computers in Biology and Medicine 66 (2015): 144-153.

[3] Polania LF, Carrillo RE, Blanco-Velasco M, Barner KE. Compressed sensing based method for ECG compression. In: IEEE international conference on acoustics, speech and signal processing 2011 May; pp. 761-764.

[4] Tamboli RR, Savkoor MA, Jana S, Manthalkar R. On the sparsest representation of electrocardiograms. In Computing in Cardiology 2013; pp. 479-482.

[5] Bendifallah A, Benzid R, Boulemden M. Improved ECG compression method using discrete cosine transform. Electron. Lett. 2011 Jan 20;47(2):87-9.

[6] Hilton ML. Wavelet and wavelet packet compression of electrocardiograms. IEEE Transactions on Biomedical Engineering. 1997 May;44(5):394-402.

[7] Lu Z, Kim DY, Pearlman WA. Wavelet compression of ECG signals by the set partitioning in hierarchical trees algorithm. IEEE transactions on Biomedical Engineering. 2000 Jul;47(7):849-56.

[8] Islam SM, Huang X, Sharma D. Wavelet based denoising algorithm of the ECG signal corrupted by WGN and Poisson noise. In International Symposium on Communications and Information Technologies 2012 Oct; pp. 165-168.

[9] Tamboli RR, Reddy DS, Jana S. A hybrid Fourier/wavelet technique for improved ECG signal approximation. In IEEE International Conference on Signal Processing, Computing and Control 2013 Sep; pp. 1-6.

[10] Goldberger AL, Amaral LA, Glass L, Hausdorff JM, Ivanov PC, Mark RG, Mietus JE, Moody GB, Peng CK, Stanley HE. Physiobank, physiotoolkit, and physionet components of a new research resource for complex physiologic signals. Circulation. 2000 Jun 13;101(23):e215-20.

Address for correspondence:

Roopak R Tamboli

513, Boys' Hostel, IIT Hyderabad,

Yeddumailaram, Telangana - 502205, India.

ee13p0008eith.ac.in 